\title{
Mediastinal malignancy: ultrasound guided biopsy through the supraclavicular approach
}

\author{
Pan-Chyr Yang, Dun-Bing Chang, Yung-Chie Lee, Chong-Jen Yu, Sow-Hsong Kuo, \\ Kwen-Tay Luh
}

\begin{abstract}
Background Malignancies located in the upper middle mediastinum usually do not have a sufficiently large acoustic window to permit a conventional ultrasound guided parasternal biopsy. This study was concerned with an alternative approach whereby ultrasound is applied through the supraclavicular paratracheal window to allow percutaneous biopsy of middle mediastinal malignancies.
\end{abstract}

Methods Fifteen patients who had upper mediastinal malignancies not in contact with the chest wall underwent real time and Doppler ultrasonographic studies by the supraclavicular approach. None of these tumours could be reached by conventional ultrasound guided parasternal biopsy. The ultrasound was scanned downwards through the supraclavicular fossa, along the acoustic window of the paratracheal soft tissue space. Percutaneous aspiration biopsy was performed with a 22 gauge needle under ultrasound guidance. If fine needle aspiration could not obtain an adequate tissue smear an 18 gauge Trucut biopsy was performed to obtain a histological diagnosis.

Results Twelve of 15 mediastinal malignancies were detected by ultrasound through the supraclavicular approach. These 12 patients underwent percutaneous needle aspiration biopsy under ultrasound guidance. Four of the patients also had a Trucut biopsy because the needle aspirates from the tumours were inadequate. The needle had to pass through the jugular veins in four patients who received fine needle aspiration but in none of the patients who required a Trucut biopsy. Definite histological diagnoses were obtained in all 12 of these patients. Ten of the tumours were malignant and two benign. None of the patients developed any complication.

Conclusions Ultrasound and ultrasound guided biopsy through the supraclavicular paratracheal window provides a new approach for malignancy located in the upper middle mediastinum, which cannot be reached by conventional ultrasound guided parasternal biopsy. The diagnostic yield of this technique is high and the procedure is relatively safe.

Percutaneous transthoracic needle biopsy under ultrasound guidance has recently been shown to be a reliable and safe diagnostic procedure for mediastinal malignancies. ${ }^{1-6}$ Even a large bore cutting biopsy can be safely performed on mediastinal tumours. ${ }^{67}$ There are several advantages for ultrasound guided biopsy for mediastinal malignancies. Ultrasound accurately localises the tumour, clearly depicts its anatomical relation to the large mediastinal vessels, and monitors the biopsy procedure in real time. ${ }^{4-6}$

Ultrasound guided mediastinal biopsy does, however, have its limitations. Conventional ultrasound guided biopsy is performed by a parasternal or intercostal approach. ${ }^{1-6}$ The tumour has to lie within the anterior or posterior mediastinum and attachment of the lesion to the chest wall must be sufficient to create a good "acoustic window" for the ultrasound beam to penetrate. ${ }^{4-8}$ For those mediastinal malignancies located in the middle mediastinum, or those lesions that do not have a sufficient acoustic window, the visualisation of the lesions by ultrasound will not be successful and ultrasound guided biopsy is not possible.

In this study we describe a new approach whereby ultrasound is applied through the supraclavicular paratracheal soft tissue space as a guide to biopsy of malignancies located within the upper mediastinum, particularly those tumours that did not have large enough acoustic windows to permit conventional ultrasound guided parasternal biopsy.

\section{Methods}

From January 1991 to August 199115 patients with mediastinal malignancy were referred for ultrasound examination and ultrasound guided biopsy through the supraclavicular approach. The criteria for referral were: (1) tumour located within the upper mediastinum; (2) tumour not attached to the anterior or posterior chest wall or to the apical pleura; (3) no histological diagnosis after bronchoscopic biopsy, transbronchial needle aspiration, or neck lymph node biopsy; (4) no coagulopathy or bleeding tendency; (5) cooperation of the patient. There were 12 patients who had lesions detectable by ultrasound and for whom ultrasound guided biopsy was considered safe. In three patients an adequate image was not obtained because the tumour was deep in the mediastinum and the acoustic window of paratracheal soft tissue space was less than $1 \mathrm{~cm}$ in width. 
Results of ultrasound guided aspiration biopsy of 12 mediastinal tumours through supraclavicular approach

\begin{tabular}{llll}
\hline $\begin{array}{l}\text { Patient No/ } \\
\text { sex/age }(y)\end{array}$ & $\begin{array}{l}\text { Lesion } \\
\text { size }(\mathrm{cm})\end{array}$ & $\begin{array}{l}\text { Lesion } \\
\text { depth }(\mathrm{cm})\end{array}$ & $\begin{array}{l}\text { Histology of aspiration } \\
\text { biopsy specimen }\end{array}$ \\
\hline $1 / \mathrm{M} / 74$ & $4 \times 5$ & $3 \cdot 0$ & Squamous cell carcinoma \\
$2 / \mathrm{M} / 55$ & $5 \times 6$ & $4 \cdot 0$ & Adenocarcinoma \\
$3 / \mathrm{M} / 70$ & $3 \times 4$ & $3 \cdot 5$ & Squamous cell carcinoma \\
$4 / \mathrm{M} / 74$ & $4 \times 4$ & $4 \cdot 0$ & Small cell carcinoma \\
$5 / \mathrm{M} / 70$ & $4 \times 5$ & $4 \cdot 0$ & Poorly differentiated carcinoma \\
$6 / \mathrm{M} / 69$ & $3 \times 4$ & $3 \cdot 5$ & Squamous cell carcinoma \\
$7 / \mathrm{F} / 65$ & $4 \times 4$ & $3 \cdot 5$ & Squamous cell carcinoma \\
$8 / \mathrm{M} / 71$ & $5 \times 5$ & $4 \cdot 0$ & Small cell carcinoma \\
$9 / \mathrm{M} / 78$ & $6 \times 5$ & $3 \cdot 5$ & Small cell carcinoma \\
$10 / \mathrm{M} / 69$ & $4 \times 5$ & $5 \cdot 0$ & Poorly differentiated carcinoma \\
$11 / \mathrm{F} / 70$ & $5 \times 6$ & $4 \cdot 5$ & Thymoma \\
$12 / \mathrm{F} / 60$ & $4 \times 5$ & $4 \cdot 0$ & Goitre \\
\hline
\end{tabular}

*Tru-cut biopsy was done for definite histological diagnosis.

Of the 12 patients who had ultrasound guided mediastinal biopsy, nine were men and three women; their ages ranged from 55 to 78 (mean 69) years. At the time of the ultrasound guided biopsy these 12 patients had already had computed tomography (Somatom DR; Siemens Medical System, Iselin, New Jersey) of the chest, which documented the location of each tumour in the middle mediastinum.

All patients were examined with real time, linear array, convex, and sector ultrasound units with $3 \cdot 75,5 \cdot 0$, and $7 \cdot 5 \mathrm{MHz}$ transducers (Aloka SSD 630 and Toshiba 100A, Tokyo). The patients lay supine. A pillow was put below the scapulae to keep the neck extended. The ultrasound transducer was scanned downward through the supraclavicular fossa, along the paratracheal soft tissue space. A convex transducer was most suitable for supraclavicular ultrasound examination. The sonographic images were recorded on Polaroid film (Polaroid, Cambridge, Massachusetts) and analysed both for evidence of tumour and for the relation of the tumour to the surrounding great vessels. Doppler ultrasound was also used to identify the arteries and veins within the supraclavicular fossa. This information was very important in guiding the percutaneous biopsy needle. For those patients with demonstrable tumours the size, shape, echogenicity, and depth of lesion were determined.

After location and demarcation of the tumour margin informed consent was obtained and the lesion was subjected to percutaneous transthoracic aspiration biopsy through the supraclavicular paratracheal soft tissue space under ultrasound guidance. The skin was prepared and a local anesthetic agent was applied. A sterile puncture probe (UST507BP) with an adjustable needle guiding channel was used to guide the percutaneous biopsy needle. A 22 gauge needle with an outer sheath and inner stylet was used for the biopsy. ${ }^{69}$ The patient was asked to hold his breath and aspiration was performed. It usually took 10 seconds to complete a procedure. The ultrasound guided aspiration biopsy technique is similar to that for percutaneous biopsy of a pulmonary nodule. ${ }^{8}$ The aspirated material was smeared on to slides and submitted for cytological and histological examination. When the cytology smear was inadequate needle aspiration was repeated. If the repeat aspiration did
Figure 1 Man aged 74 years (patient 1) with a tumour in the right upper mediastinum. A-Chest radiograph. B-Ultrasound scan from the right paratracheal space showing a $4 \times 5 \mathrm{~cm}$ hypoechoic tumour deeply seated in the upper mediastinum (arrow heads). There are numerous vessels in the supraclavicular fossa.

Arrows indicate the vessels in the soft tissue of the supraclavicular fossa. CComputed tomography scan at the level of the second thoracic spine showing that the tumour did not extend to the chest wall and apical pleura. The ultrasound is scanned through the paratracheal soft tissue space (arrow). D-Computed

tomography scan at the level of third thoracic spine showing a tumour in the right paratracheal region (arrow head). Ultrasound guided biopsy of this patient showed squamous cell carcinoma.
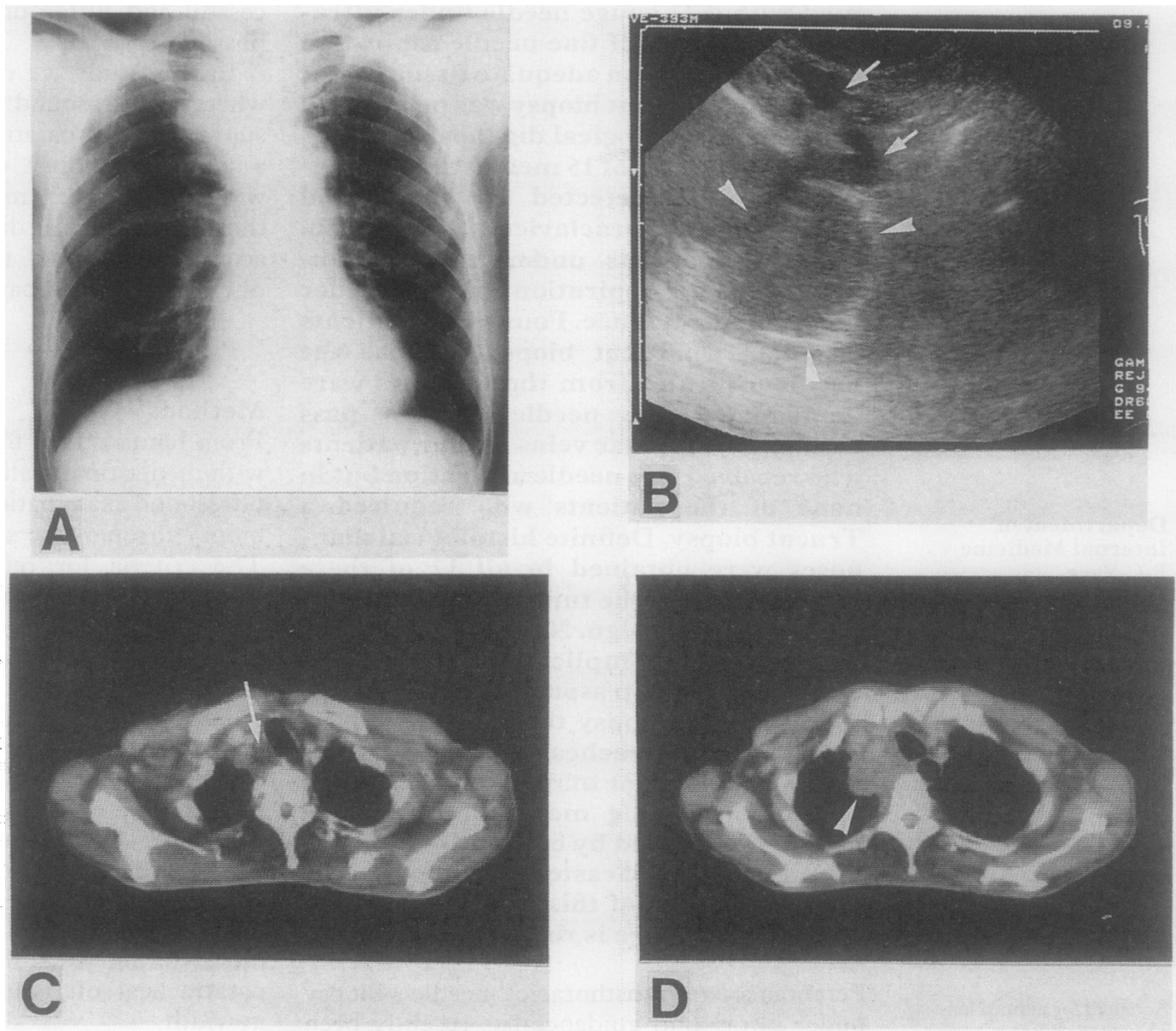
Figure 2 Man aged 55 years (patient 2) with a tumour in the left upper mediastinum. A-Chest radiograph. B-Computed tomography scan showing that the tumour is in the middle mediastinum, which is not in contact with the chest wall (arrow head). There is a central necrotic cavity. C-Ultrasound scan through the supraclavicular approach showing $5 \times 6 \mathrm{~cm}$ hypoechoigenic tumour in the upper mediastinum (arrow heads) with a cavity (arrow).

Ultrasound guided aspiration biopsy showed adenocarcinoma.
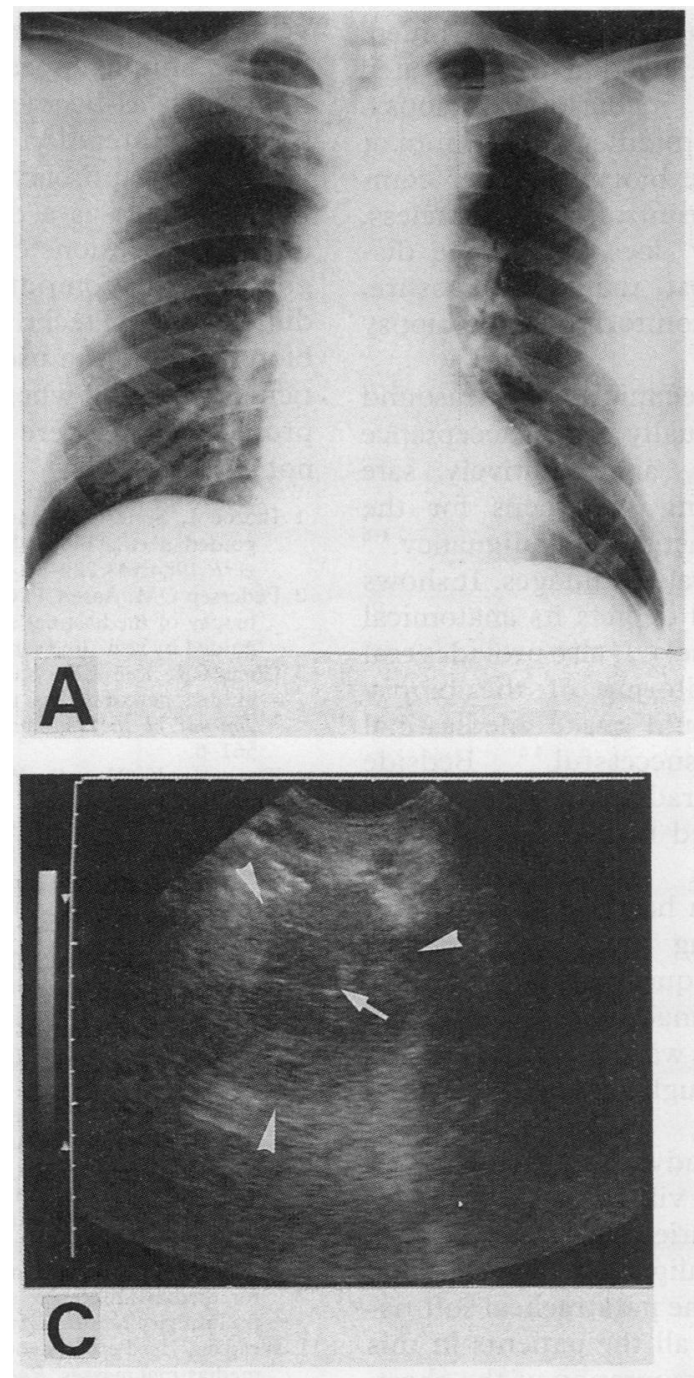

not obtain an adequate smear of tissue and if a sufficient window width was available, an 18 gauge Trucut biopsy (Top Surgical, Tokyo) was performed to obtain the histological diagnosis. ${ }^{6}$ After needle aspiration the patient had a routine chest radiograph taken.

\section{Results}

Twelve patients who had a tumour in the upper mediastinum had successful biopsies under ultrasound guidance through the supraclavicular approach. The table summarises the size and depth of the lesions and the cytological appearances of ultrasound guided biopsy specimens. Tumour size as measured by ultrasound ranged from $3 \times 4$ to $6 \times 6 \mathrm{~cm}$. All of the tumours were visible on the chest radiographs. The depth of the lesions within the mediastinum ranged from 3.0 to $5.0 \mathrm{~cm}$ with a mean of 3.9 (SD 0.5$) \mathrm{cm}$. The biopsy route was preselected on the basis of ultrasound images to avoid penetrating vessels. If penetrating the vessels was unavoidable only the arteries detected by Doppler ultrasound were avoided. The needle had to pass through the jugular vein in four patients whereas the Trucut biopsy needle did not enter any great vessel. Two tumours had one aspiration, eight had two, and two had three aspirations. Four tumours also underwent Trucut biopsy for histological diagnosis.

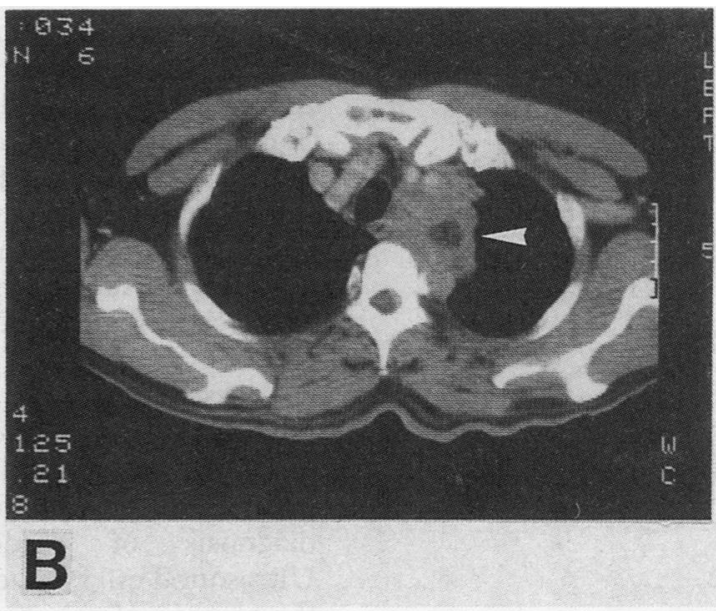

Ten of the tumours were diagnosed by needle aspiration biopsy as malignant and two as benign. Three were small cell carcinomas, four squamous cell carcinomas, one an adenocarcinoma, two poorly differentiated carcinomas, one a thymoma, and one a goitre. Two small cell carcinomas, one squamous cell carcinoma, and the thymoma also had histological confirmation from Trucut biopsy.

Figure 1 shows a tumour in the upper mediastinum of a 74 year old man (patient 1 ). Computed tomography of the chest showed that the tumour was not in contact with the anterior or posterior chest wall, which meant that the tumour could not be approached by parasternal biopsy. Ultrasound examination from the supraclavicular approach and with scanning through the paratracheal soft tissue space showed a hypoechoic tumour in the upper mediastinum. There were prominent vessels in the supraclavicular fossa. The needle aspiration biopsy specimen had the cytological appearance of squamous cell carcinoma.

Figure 2 shows a tumour in the upper mediastinum of a 55 year old man (patient 2), which was not in contact with the chest wall. Computed tomography showed a mediastinal tumour with central necrosis. Ultrasound scanning from the supraclavicular fossa showed a hypoechogenic tumour with cystic changes and the biopsy specimen obtained through the paratracheal window was an adenocarcinoma.

None of the patients developed haemoptysis, pneumothorax, or a local haematoma after the procedure.

\section{Discussion}

Percutaneous needle biopsy of mediastinal malignancies under fluoroscopic guidance was first described by Nordenström in $1967 .{ }^{10}$ This technique has become established as a reliable and safe procedure for mediastinal masses. ${ }^{10-14}$ Accidental puncture of large mediastinal arteries may, however, occur occasionally under fluoroscopic guidance. ${ }^{415}$ Since 1980 computed tomography has largely replaced the fluoroscopy as a guide for mediastinal biopsy. ${ }^{14-16}$ Computed tomography has the advantage of accurate localisation of the lesion and with contrast can show the relation of this to the great vessels; thus the hazard of punctur- 
ing vessels in the mediastinum can be avoided. There is no spatial restriction of computed tomography guided mediastinal biopsy. Tumour located in the middle mediastinum or perihilar region can be biopsied under computed tomography control. Nevertheless, computed tomography does have some disadvantages, such as cost, radiation exposure, and lack of real time monitoring of the biopsy procedure. $^{4}$

In recent years the technique of ultrasound guided biopsy has gradually gained acceptance as a useful, reliable, and relatively safe procedure for obtaining specimens for the diagnosis of mediastinal malignancy. ${ }^{1-6}$ Ultrasound offers several advantages. It shows the tumour clearly and depicts its anatomical relation to the great vessels. It also provides real time continuous monitoring of the biopsy procedure, which should make mediastinal biopsy safe and successful. ${ }^{46}$ Bedside availability and lack of radiation exposure also make ultrasound guided biopsy a convenient procedure.

The major limitation hampering the use of ultrasound for guiding mediastinal biopsy was the frequent inadequacy of the "acoustic window." The mediastinal tumour has to be in contact with the chest wall and the attached area must be large enough for the ultrasound beam to penetrate. ${ }^{4-6}$

In this small series study we have shown that ultrasound examination via the supraclavicular approach, with the patient lying with neck extended, can reveal malignancy of the middle mediastinum through the paratracheal soft tissue window. Although all the patients in this study had computed tomography of the chest, this is not necessary for performing an ultrasound guided biopsy. In conjunction with Doppler ultrasound, we were also able to differentiate the great arteries and veins. Ultrasound clearly delineates the tumour, distinguishing it from the surrounding great vessels, and so allows a biopsy route to be preselected to avoid the major arteries. If penetrating vessels is unavoidable, penetration of the neck veins by a fine needle of 22 gauge is acceptable. The technique appears safe and no complications such as haemorrhage or pneumothorax were encountered in this study.

This new approach extends the applicability of ultrasound as a diagnostic tool. Although mediastinal lesions can be biopsied under the guidance of computed tomography without difficulty, ${ }^{16}$ the technique of ultrasound guided biopsy provides a useful alternative. It is particularly helpful where patients have transport problems and where computed tomography is not available.

1 Ikezoe J, Sone S, Higashihara T, et al. Sonographically guided needle biopsy for diagnosis of thoracic lesions. guided needle biopsy

2 Pedersen OM, Aasen T, Gulsvi KA. Fine needle aspiration biopsy of mediastinal and peripheral pulmonary masses guided by real-time sonography. Chest 1986;89:504-7.

3 Chen CR, Lee LN, Kuo SH, Luh KT. Ultrasonically guided percutaneous mediastinal cytology and biopsy. Journal of the Formosan Medical Association 1986;85: 561-6.

4 Wernecke K, Vassallo P, Peters PE, van Bassewitz DB Mediastinal tumors: biopsy under US guidance. Radiology 1989;172:473-6.

5 Saito T, Kobayashi H, Sugama Y, Tamaki S, Kawai T, Kitamura $S$. Ultrasonically guided needle biopsy in the diagnosis of mediastinal masses. Am Rev Respir Dis 1988; diagnosis of

6 Yu CJ, Yang PC, Chang DB, et al. Evaluation of ultrasoundguided biopsies of mediastinal masses. Chest 1991;100. 399-405.

7 Yang PC, Lee YC, Yu CJ, et al. Ultrasound guided biopsy of thoracic tumours: a comparison of large bore cutting biopsy with fine needle aspiration. Cancer (in press).

8 Yang PC, Luh KT, Wu HD, et al. Lung tumors associated with obstructive pneumonitis: US studies. Radiology 1990;174:717-20.

9 Yang PC, Luh KT, Shen JC, Kuo SH, Yang SP. Peipheral pulmonary lesions: ultrasonography and ultrasonically guided aspiration biopsy. Radiology 1985;155:451-6.

10 Nordenström B. Paraxiphoid approach to the mediastinum for mediastinography and mediastinal needle biopsy: a preliminary report. Invest Radiol 1967;2:141-6.

11 Westcott JL. Percutaneous needle aspiration of hilar and mediastinal masses. Radiology 1981;141:323-9.

12 Todd TRJ, Weisbrod G, Tao LC, et al. Aspiration needle biopsy of thoracic lesions. Ann Thorac Surg 1981;32: $154-60$.

13 Adler OB, Rosenberger A, Peleg $\mathrm{H}$ : Fine-needle aspiration biopsy of mediastinal masses. AJR 1983;140:893-6.

14 Weisbrod GL, Lyons DJ, Tao LC, Chamberlain DW. Percutaneous fine-needle aspiration biopsy of mediastinal lesions. AJR 1984;143:525-9.

15 Westcott JL. Percutaneous transthoracic needle biopsy. Radiology 1988;169:593-601.

16 van Sonnenberg E, Casola G, Ho M, et al. Difficult thoracic lesions: CT-guided biopsy experience in 150 cases. Radiology 1988;167:457-61. 